\title{
Collective behavior in nuclear interactions and shower development
}

\author{
V. Canoa ${ }^{1}$, N. Armesto ${ }^{2}$, C. Pajares ${ }^{1}$ and R. A. Vázquez ${ }^{1}$ \\ 1 Departamento de Física de Partículas, \\ Universidade de Santiago de Compostela, \\ E-15706 Santiago de Compostela, Spain and \\ 2 Department of Physics, CERN, Theory Division, CH-1211 Genève 23, Switzerland
}

\begin{abstract}
The mechanism of hadronic interactions at very high energies is still unclear. Available accelerator data constrain weakly the forward rapidity region which determines the development of atmospheric showers. This ignorance is one of the main sources of uncertainty in the determination of the energy and composition of the primary in hadron-induced atmospheric showers. In this paper we examine the effect on the shower development of two kinds of collective effects in high-energy hadronic interactions which modify the production of secondary particles. The first mechanism, modeled as string fusion, affects strongly the central rapidity region but only slightly the forward region and is shown to have very little effect on the shower development. The second mechanism implies a very strong stopping; it affects modestly the profile of shower maximum but broadens considerably the number distribution of muons at ground. For the latter mechanism, the development of air showers is faster mimicking a heavier projectile. On the other hand, the number of muons at ground is lowered, resembling a shower generated by a lighter primary.
\end{abstract}

PACS numbers: 13.85.Tp, 13.85.-t, 96.40.De 


\section{INTRODUCTION}

The cosmic ray energy spectrum extends up to several $10^{20} \mathrm{eV}$. The presence of these high energy particles (UHECRs) poses an enigma, since many good arguments suggest that they should not be observed because of the GZK cutoff [1]. This apparent contradiction has stimulated a variety of more exotic explanations of their origin and composition which is usually known as the GZK puzzle. Both theoretically and experimentally the problem has been the subject of considerable efforts [2].

One of the major uncertainties is the lack of information about the composition of the highest energy cosmic rays. Besides being a difficult, indirect measurement with contradictory experimental results, it involves extrapolations of hadronic physics models far beyond the actual measurements. The systematic effects due to the uncertainty of the hadronic interactions, see e.g. [3], at these energies are far from being established.

In Cosmic Ray Physics, it is usually assumed that a nucleus-nucleus collision occurs as an independent combination of nucleon-nucleon interactions. This approximation is useful in that it allows to obtain scaling relations for shower properties like shower maximum and the muon content, which are roughly obeyed. But it is not justified at high energies: a beam nucleon may interact several times with different target nucleons, which will invalidate the approximation trivially. A more interesting possibility would be the existence of a higher degree of collectivity (defined in the sense of a failure in the independence of the superposition of nucleon-nucleon interactions). This collectivity should be present in any type of hadronic collisions, but its enhancement due to the hadron size makes it more prominent for nucleusinduced reactions. It could eventually lead to the formation of new nuclear phases during the collision, like the Quark-Gluon Plasma. The existence or not of such new form of matter at the SPS at CERN and RHIC at BNL has been widely discussed [4]. For example, it is of relevance for our study that some violation of the linear scaling law for the rapidity shift of the projectile nucleons, which works at lower energies, has already been observed at RHIC [5].

Here we will investigate the effects of collective behavior on the development of high energy cosmic ray showers. Collective behavior may affect shower development in two different ways. First, it could lead to changes in the total cross sections. Such changes have been investigated in some models and found to be negligible, see e.g. [6]. Second, it is generally 
expected that any collective behavior would affect the spectra of secondary particles; this is the point that we will try to address in our study, exploring it in two directions. On the one hand, it may reduce the total multiplicity due to shadowing. As we will see below this would affect the shower development only if the multiplicity reduction can be carried over the whole cascade process [7, 8]. This is, in general, not expected. Alternatively, collective effects may affect the secondary particle spectrum, in particular in the large Feynman- $x$ region, thereby modifying the inelasticity of the collision. This may have important consequences in shower development as we will show below. Other possible consequences of collectivity like heavy flavor production or modifications of large- $p_{t}$ particle production are expected to have much smaller effect on the shower development.

We will analyze the second possibility within both directions. We will consider first the possibility of string fusion [9, 10] as a collective effect which strongly reduces the multiplicities in the small Feynman- $x$ region but modifies only slightly the large Feynman- $x$ one. It turns out that this effect produces negligible modifications in shower development, as expected. We then consider a second possibility in which extremely strong collective effects do modify the stopping power and inelasticity in the collision. Concretely, it has been suggested recently by Mishustin and Kapusta [11] that in the central region of a high-energy collision, strong chromo-electric fields are formed. They will attract the forward and backward particles, reducing considerably the rapidity of the fast-moving, leading secondaries. This effect is expected to increase the inelasticity even for peripheral collisions. It will lead to a small change in the shower depth profile but it will induce a large broadening of the number distribution of muons at ground.

The plan of the paper is as follows. In the next Section we will briefly discuss the ingredients present in hadronic models introduced in the simulation of atmospheric showers. In Section III we will analyze the influence of the string fusion mechanism on shower development. The extreme stopping scenario of [11] will be discussed in Section IV, and Section V will be devoted to our conclusions.

\section{HADRONIC MODELS}

Most of the models which simulate interactions of nucleons are based on the Gribov-Regge Theory (GRT) [12], which most successfully describes elastic scattering and, via the optical 
theorem, the total hadronic cross section as a function of energy. In GRT the observed rise of the cross section at high energies is a consequence of the exchange of multiple supercritical Pomerons, the Pomeron being here the effective exchange. Inelastic processes are described by cut Pomerons. In the Dual Parton Model (DPM) or Quark-Gluon String Model (QGSM) 13], each cut Pomeron leads to two color strings which fragment subsequently into colorneutral hadrons. The number of exchanged strings (or Pomerons) rises with energy. Most models widely used in Cosmic Ray Physics to compute hadronic collisions at high energies, e.g. 14, 15, 16], are based on these ideas for the low transverse momentum region; for hard particle production, perturbative QCD is used with a matching between the soft and hard components.

A possible formulation of GRT can be done 17] assuming that strings have a transverse size $A=\pi r^{2}$. As the number of strings increases with collision energy, mass number of projectile and target and decreasing impact parameter (increasing centrality) of the collision, it is expected that the strings overlap and can no longer be considered to evolve and fragment independently. The simplest possibility to consider is fusion of strings. The fusion probability is determined by some parton or string transverse dimension and by the corresponding parton-parton cross section. This model is the string fusion model (SFM) [9] embedded in the DPM. In the last version of this model, in the form of the program PSM 10], only fusion of pairs of strings is taken into account and consecutive fusions of strings are neglected. Therefore, the string fusion parameter, the transverse area occupied by a string, is considered as an effective parameter that is taken to be [10] $\sigma_{p}=2 \pi r^{2}=7.5 \mathrm{mb}$. Let us note that for proton-nucleus collisions the number of strings is much reduced compared to nucleus-nucleus collisions. Therefore even at the highest cosmic ray energies the effect of string fusion on proton-air collisions is very small.

Consequences of string fusion [9, 10] are the reduction of multiplicities at central rapidities, a slight increase in the transverse momentum of the produced particles, enhanced production of strangeness and of baryon/antibaryons and enhanced correlations between particles produced in the forward and backward rapidity hemispheres. Nevertheless, these effects are mainly noticeable at central rapidities, the very forward and very backward regions being only slightly affected [18]. Results of the program PSM for cross sections, multiplicities, longitudinal and transverse momentum spectra and production of different hadron species can be found in [10], together with an extensive comparison to accelerator 


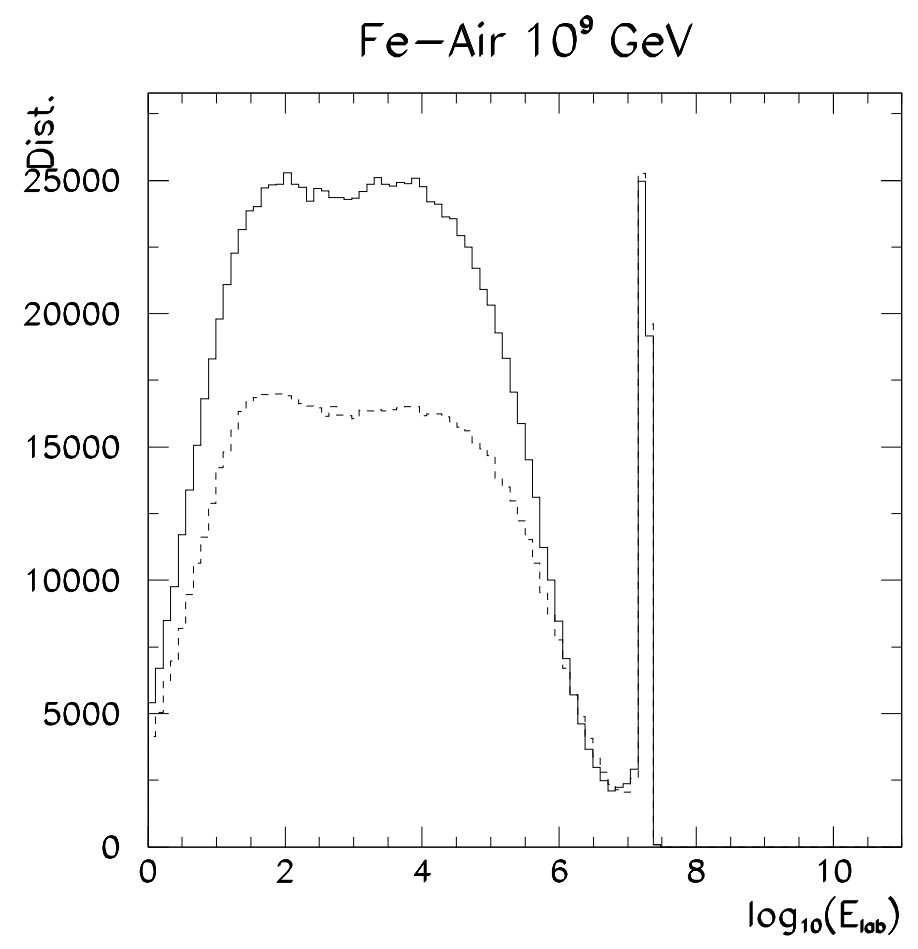

FIG. 1: Secondary particle energy distribution for Iron-Nitrogen collisions at $10^{9} \mathrm{GeV}$ laboratory energy without fusion (continuous line) and with fusion (dotted line).

data. In Fig. [1 the energy distribution for secondary particles in Iron-Nitrogen collisions at $10^{9} \mathrm{GeV}$ laboratory energy is shown with and without fusion. The reduction of multiplicities for low-energy secondaries due to fusion is clearly seen, while the distribution of high-energy secondaries is very modestly affected.

An appealing possibility to extend the mechanism of string fusion, allowing for a truly collective phase, is to consider a percolation phase transition [19]: When the density of strings in the transverse space of the collision reaches a critical value, paths of overlapping strings crossing the total available area appear and the average cluster size increases suddenly with increasing string density. Consequences of percolation have been studied extensively 20]. This phenomenon implies the existence of very strong chromo-electric fields stretched between projectile and target color sources, like in the model of [11] which will be discussed in Section IV.

As a last comment in this Section, let us note that fusion and percolation of strings can be viewed as effective realizations of a collective mechanism of interaction between the exchanged Pomerons, as those introduced in [15, 16]. This mechanism also leads to a 
reduction of multiplicities.

\section{AIR SHOWERS AND STRING FUSION}

We have implemented the PSM with/without string fusion (program PSM-1.0 [10]) in the Air shower simulation program AIRES [21]. In our implementation, the possibility of string fusion is considered only for the first nucleus-nucleus collision. This is a valid approximation since the energy of the secondary collisions is greatly reduced compared to the initial energy and also because these secondary collisions take place mainly between individual nucleons and mesons and air nuclei; thus, the density of strings is reduced in the secondary collisions making the probability of fusion negligible. The AIRES hadronic model only takes into account kaons, nucleons, and pions. All the other hadronic particles produced in the PSM (or QGSJET [14]) are forced to decay before being passed to the AIRES subroutines.

We have run our code for iron initiated showers with energies from $10^{14}$ to $10^{20} \mathrm{eV}$ and with a relative thinning energy (see [21]) of $10^{-6}$. 500 showers for each energy have been generated both with and without string fusion.

Our results can be seen in Fig. 2,3, In Fig. 2 we show the $X_{\max }$ distribution for showers of $10^{20} \mathrm{eV}$, with and without fusion. The difference in the average value of the $X_{\max }$ is less than $0.6 \%$, compatible with a null result, despite the fact that at these energies the difference in multiplicity of the first collision is about a $40 \%$. The average shower development are indistinguishable, as can be seen in Fig. 2 for the longitudinal development of electrons and in Fig. 3 for that of the muons.

These results seem to contradict previous estimations of the effect [7, 8] and also the elongation rate theorem [22]. However both the elongation rate theorem and the calculations of references [7, 8] assumed that the reduction of multiplicity can be carried over the whole cascade process. For processes like the one discussed here in which the reduction of multiplicity is related to the density of interacting strings, this is not expected. From this result, we conclude that string fusion (or any collective effect which affects only the central rapidity region) has no or very small effect on the shower development. 


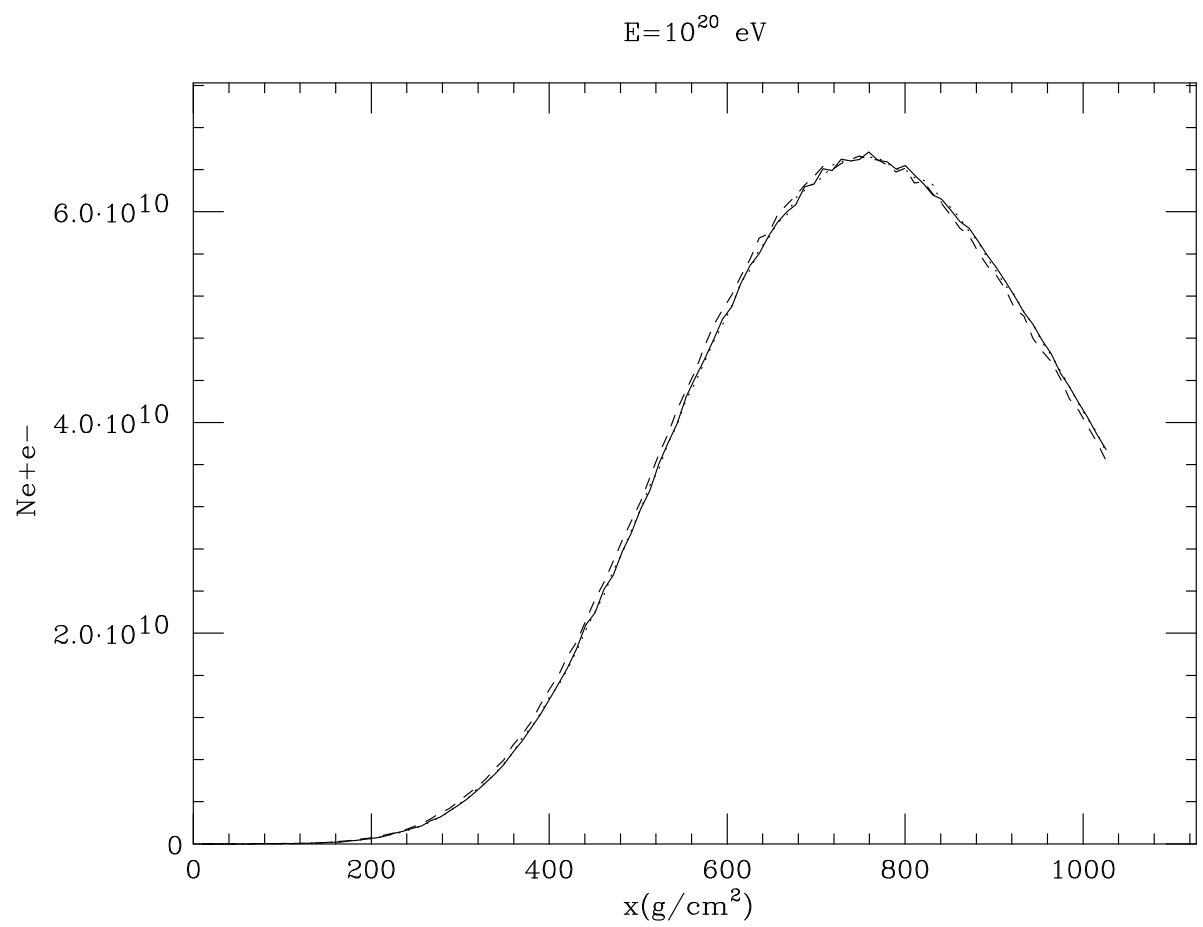

FIG. 2: Average $e^{+} e^{-}$longitudinal development for iron showers of $10^{20} \mathrm{eV}$ in the case with fusion (dashed line) and without fusion (continuous line); also results from QGSJET are shown (dotted line).

\section{AIR SHOWERS AND REDUCTION OF PARTICLE PRODUCTION IN THE FRAGMENTATION REGION}

A different possibility that produces a large stopping as a consequence of strong collective effects, was recently considered by Mishustin and Kapusta 11]. In the center-of-mass frame a nuclear collision can be viewed as two thin, Lorentz-contracted, slabs of nucleons colliding and going one through each other. The collision proceeds through the exchange of color between nucleons of the projectile and target. After the collision the two slabs of nucleons will move fast apart, and in the central region a strong coherent chromo-electric field, modeled as color strings stretched between the excited color charges in the slabs, may form. The backreaction of this field on the slabs will decelerate them, causing a large stopping power. The situation resembles the movement of charged capacitor plates on the electric field created by themselves, with a conversion of kinetic into potential energy. This deceleration will continue until the field lines stretch so much that they decay into quark-antiquark pairs 


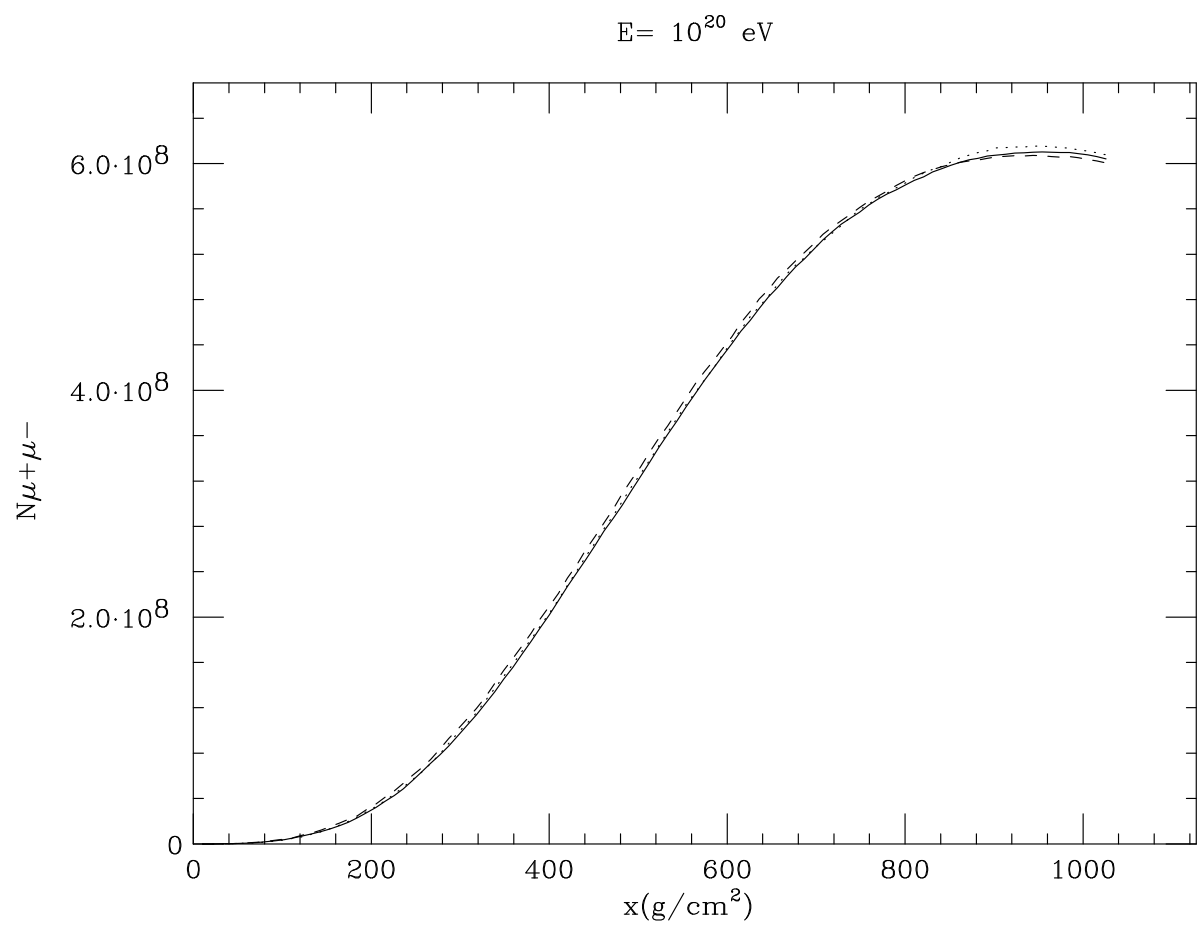

FIG. 3: Average $\mu^{+} \mu^{-}$longitudinal development for iron showers of $10^{20} \mathrm{eV}$, with the same line convention as in Fig. 2 ,

and gluons produced from the vacuum via the Schwinger mechanism, which will eventually neutralize the chromo-electric field.

The resulting (projectile or target) slab will have a final $\gamma$-factor given by [1]]

$$
\gamma^{*}=\cosh y^{*}=\gamma_{0}\left[1-\frac{\tau_{0}}{\lambda}\left(v_{0} \sqrt{1+\frac{\tau_{0}^{2}}{4 \lambda^{2}}}-\frac{\tau_{0}}{2 \lambda}\right)\right],
$$

where $\gamma_{0}=\cosh y_{0}, v_{0}=\tanh y_{0}$ are respectively the original $\gamma$-factor and velocity of the slab and $\tau_{0}$ is the time from the start of the collision until the end of the deceleration process. $\lambda$ is the characteristic deceleration length

$$
\lambda=\frac{\epsilon \rho_{0}}{\epsilon_{f}} l,
$$

where $\epsilon$ is the initial energy per baryon, $\epsilon_{f}$ is the energy density of the chromo-electric field, $l$ is the slab thickness depending on the position in transverse plane (impact parameter), and $\rho_{0}$ is the nuclear density $\left(\rho_{0} l\right.$ being the nuclear profile function normalized to the mass number A). Following Reference [11] we parameterize the energy density on the chromo- 
electric field by

$$
\epsilon_{f}=\epsilon_{0}\left(\frac{s}{s_{0}}\right)^{\alpha / 2}\left(\frac{N_{p} N_{t}}{N_{0}^{2}}\right)^{\beta},
$$

where $\alpha \simeq 0.3$ is given by the low- $x$ structure function behavior reflecting the expected increase in the number of produced partons, and $\epsilon_{0}$ sets the energy scale. $s$ is the total center-of-mass energy squared and $s_{0}=1 \mathrm{GeV}^{2}$ is a reference energy squared. $N_{p, t}$ is the participant nucleon density of projectile and target, depending on the impact parameter of the collision, and $N_{0} \simeq 0.4 \mathrm{fm}^{-2}$. The amount of stopping power depends on the initial energy and also on the impact parameter. It is expected that $\epsilon_{f}$ will be proportional to the number of binary parton collisions, which here is parameterized through the dependence on the product $N_{p} N_{t}$. For uncorrelated collisions $\beta \simeq 1$, while in the case of strong correlations (like those assumed in percolation of strings [20]) one expects $\beta \simeq 0.5 \div 0.7$.

In this model the results depend on the product $\epsilon_{0} \tau_{0}$. With these parameters chosen such that $\left\langle\epsilon_{f}\right\rangle \tau_{0} \simeq 6 \mathrm{GeV} / \mathrm{fm}^{2}$ for central $\mathrm{Au} \mathrm{Au}$ collisions at center-of-mass energy $65 \mathrm{GeV}$ per nucleon, one gets a final center-of-mass energy of $3.5 \mathrm{GeV}$ per baryon [11]. This can explain the strong stopping power already observed at RHIC [5].

The participant nucleon density $N_{p}, N_{t}$ depends on the impact parameter of the collision. The overlapping area of the collision is calculated integrating the profile function of the colliding nucleus. We parameterize the nuclear density using a 3 parameter Fermi distribution taken from [23].

The reduction in rapidity can be seen in Fig. 4 where we show the $\gamma$-factor of the secondary nucleons $\left(\cosh y^{*}\right)$ as a function of impact parameter for Iron-Nitrogen collisions at energy $\sqrt{s}=200 \mathrm{AGeV}$. As can be seen in the figure, for small impact parameters the reduction factor is large. For central collisions we obtain that nucleons loose more than 60 $\%$ of their energy at $E_{\mathrm{lab}}=10^{17} \mathrm{eV}$ and more than $80 \%$ for $10^{19} \mathrm{eV}$.

The effect can be further seen in Fig. 5 where we show the same $\gamma$-factor as a function of the energy for fixed impact parameter. This energy loss is compatible with the measurements for $\mathrm{Au} \mathrm{Au}$ collisions made at BRAHMS [5].

In Fig. 6] we show the center-of-mass $x_{F}$ distribution for Fe-Air collisions at $10^{18} \mathrm{eV}$ in the case of increase and with no increase of the stopping power. The forward and backward peaks are clearly reduced.

We have implemented this increasing of the stopping power in AIRES [21]. In a given 


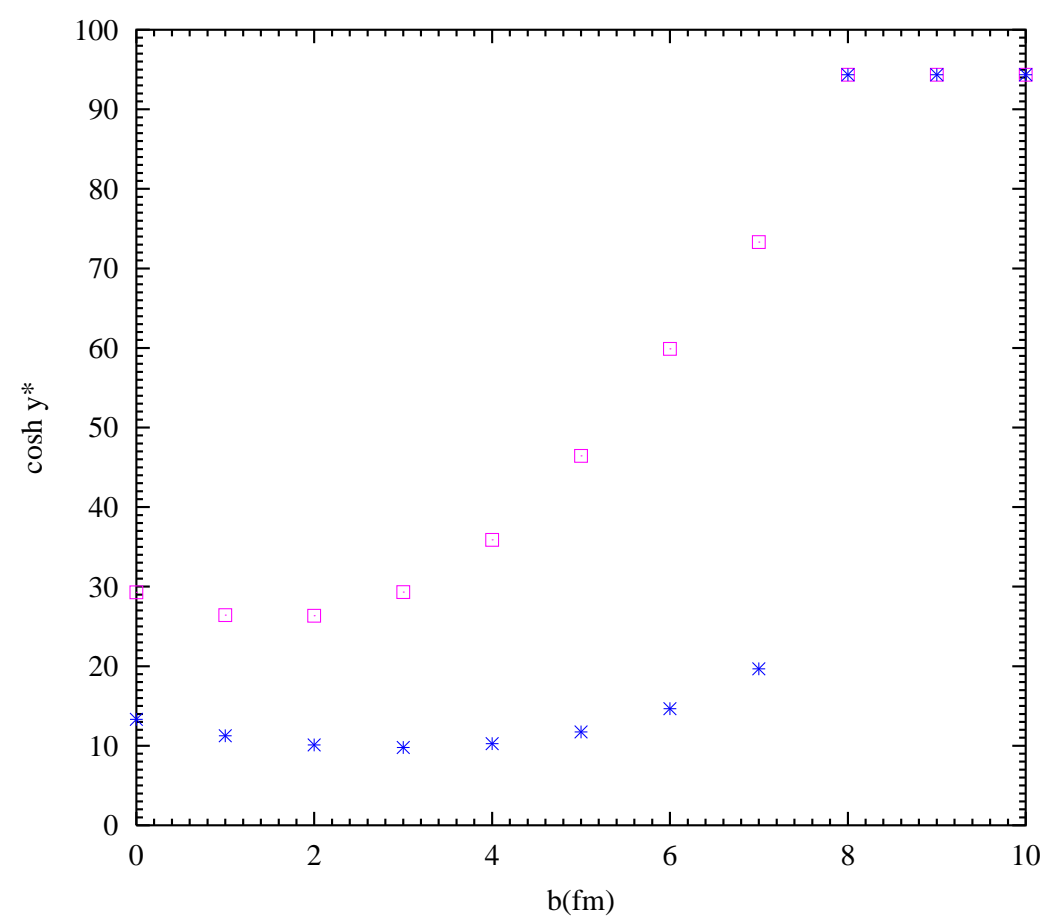

FIG. 4: Forward secondary nucleon $\gamma$-factor $\left(\cosh y^{*}\right)$ as a function of the impact parameter for Fe-Air collisions at center-of-mass energy $\sqrt{s}=200 \mathrm{GeV}$ per nucleon for the case $\beta=1$ (squares) and $\beta=0.5$ (asterisks).

nucleus-Air interactions, the impact parameter is chosen randomly and the stopping power is calculated according to Eq. 1. The collision is generated with a conventional Monte Carlo generator (QGSJET and Sibyll are implemented in AIRES). The energy (and momentum) of the leading particles obtained in the collision is reduced to match the stopping power predicted and this energy, taken from the leading particles, is redistributed between the non-leading particles. Two different version have been essayed, which probably bracket the possible range of these models: in version $\mathrm{K} 1$ we have made the reduction for all nucleons; in version $\mathrm{K} 2$, the reduction has been performed on only the participant nucleons of each nuclei. For the rest of the showering process the collisions are calculated normally, so that only the first nucleus-nucleus collision is modified. For very high energy, $E>10^{17} \mathrm{eV}$, and small impact parameters, $b<6 \mathrm{fm}$, the inelasticity increase due to this mechanism is so high that the first collision is indistinguishable from a proton-air collision in terms of inelasticity. This has the effect of modifying slightly the average $X_{\max }$ of the shower and the total number of muons. 


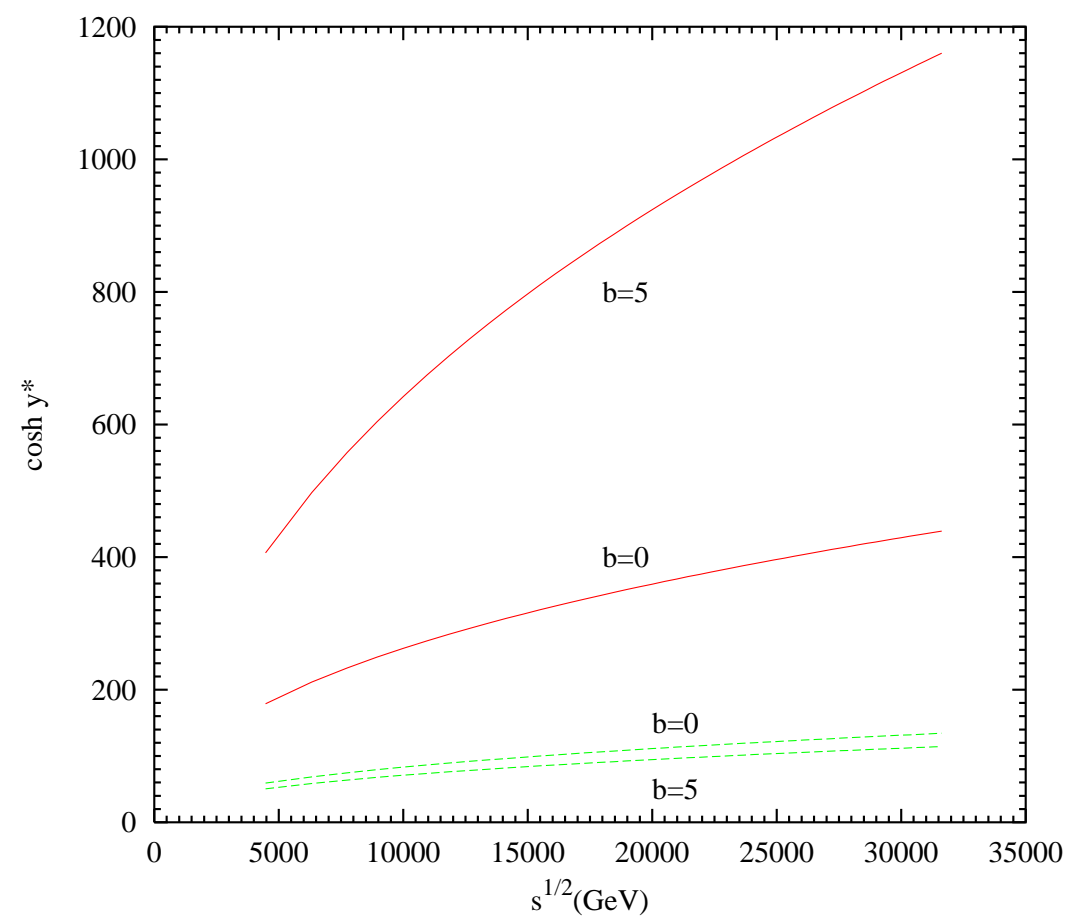

FIG. 5: Forward secondary nucleon $\gamma$-factor $\left(\cosh y^{*}\right)$ as a function of the center-of-mass collision energy per nucleon for Fe-Air collisions at fixed impact parameter (in fermi as marked) and $\beta=1$ (upper curves) and $\beta=0.5$ (lower curves).

In Fig. [7we show the distribution of shower maximum for $10^{18} \mathrm{eV}$ iron showers. Although the average value of $X_{\max }$ does not change much, $\left(\sim 13 \mathrm{~g} / \mathrm{cm}^{2}\right.$ for the $\mathrm{K} 1$ case and $\sim 8$ $\mathrm{g} / \mathrm{cm}^{2}$ for the $\mathrm{K} 2$ case) the distribution broadens, almost a factor 1.5 for the $\mathrm{K} 1$ case. This is mainly due to the dependence of the strength of the elasticity suppression on the impact parameter. To illustrate this point we show in Fig. 8 the correlation between the average $X_{\max }$ and the impact parameter of the collision. Collisions with a small first-interaction impact parameter produce showers with a lower $X_{\max }$. Due to the high degree of inelasticity of these collisions, these showers develop faster. The effect is further enhanced in the model of increased stopping power in which the created fields are large and the stopping power is also large. At large impact parameter the effect of this mechanism is negligible and the result with and without the modified stopping power is the same. In this way the correlation between the first-interaction impact parameter and $X_{\max }$ is increased as can be seen in the figure.

Finally let us turn to muon distributions. In Fig. 9 we show the muon number distribution 


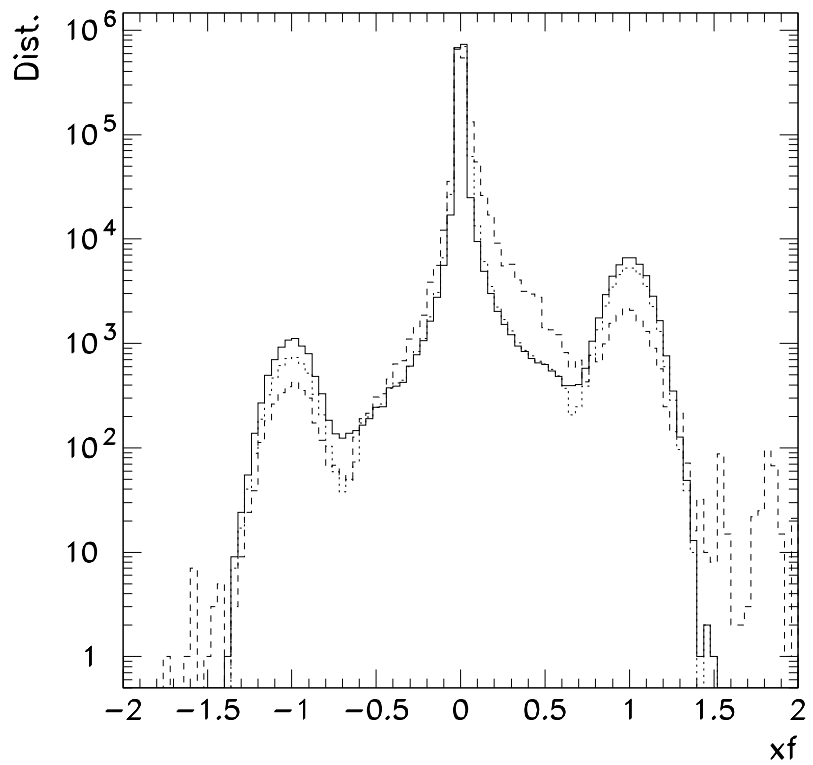

FIG. 6: Center-of-mass Feynman $x$ distribution of secondary particles in Fe-Air collisions at $10^{18}$ $\mathrm{eV}$ for the standard QGSJET model (continuous line), K1 (dashed line) and K2 (dotted line).

at ground. Here a dramatic effect is observed for showers suffering from this effect or not, the number of muons resembling that of a proton shower or a regular iron shower respectively. Both the average value and the RMS of the distribution change appreciably. In the K1 scenario the average number of muons is $7.3 \times 10^{6}$ compared to $8.6 \times 10^{6}$ for QGSJET ironinitiated showers. As a comparison, the average value of the number of muons at ground for showers initiated by protons is $7.4 \times 10^{6}$. In the K2 scenario the change in the distribution of muons at ground is small.

In Figure 10 we show the average longitudinal development for the muon component in the standard QGSJET and K1 and K2 cases. The muon component for the K1 and K2 cases is retarded with respect to the standard QGSJET.

So we observe two opposite effects of the increased stopping scenario. On the one hand, the shower development is faster than in conventional scenarios, which makes the shower look as initiated by a heavier primary (i.e. more Fe-like). On the other hand, the number of muons at ground is smaller, which makes the shower look as initiated by a lighter projectile (i.e. more proton-like). This distorts the $N_{\mu}-X_{\max }$ relation used conventionally to 


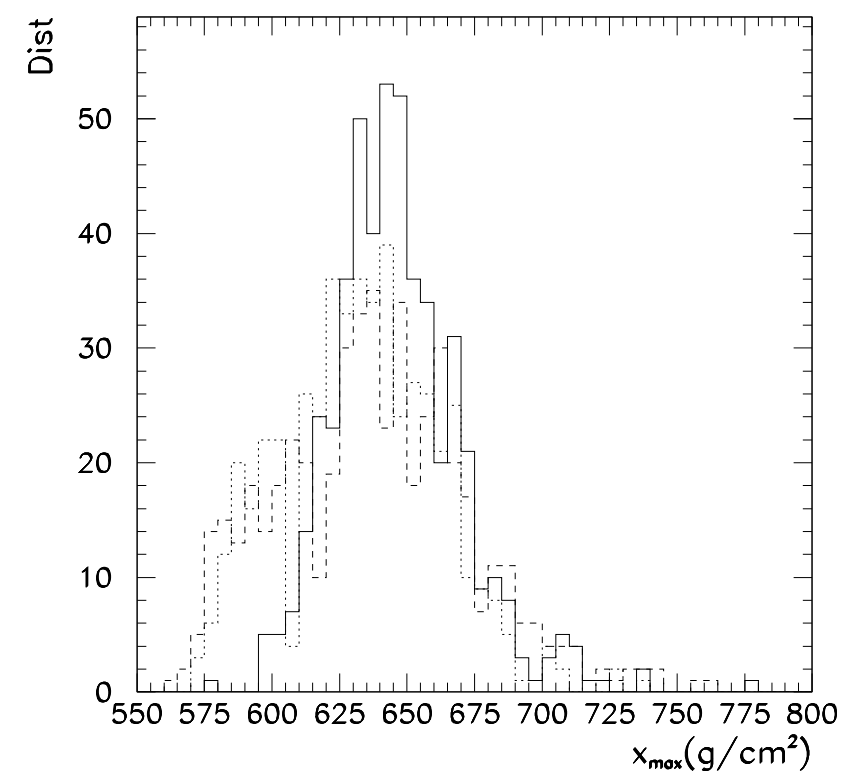

FIG. 7: Shower maximum distribution for Iron showers at $E=10^{18} \mathrm{eV}$ for standard QGSJET (continuous line), K1 (dashed line) and K2 (dotted line).

discriminate composition in an (possibly) energy dependent way. Also it introduces a source of systematics in the energy determination based on the muon number such as inclined showers. The effect may be as large as the proton-Iron difference in the number of muons.

\section{CONCLUSIONS}

The mechanism of hadronic interactions at very high energies is still uncertain. Existing experimental data refer to the central rapidity region, and the forward region is very weakly constrained. This uncertainty constitutes one of the main sources of systematic error in the study of air showers produced by UHECRs, thus limiting the energy and composition determination crucial to clarify their origin. In this paper we have tried to study the consequences on shower development of the existence of collective mechanisms in nucleus-nucleus collisions which modify secondary particle production. These mechanisms have been proposed 9, 11, 19, 20] to explain several experimental facts in heavy ion collisions. We have tried two extreme scenarios which, in our view, contain the most plausible extreme possibilities: 


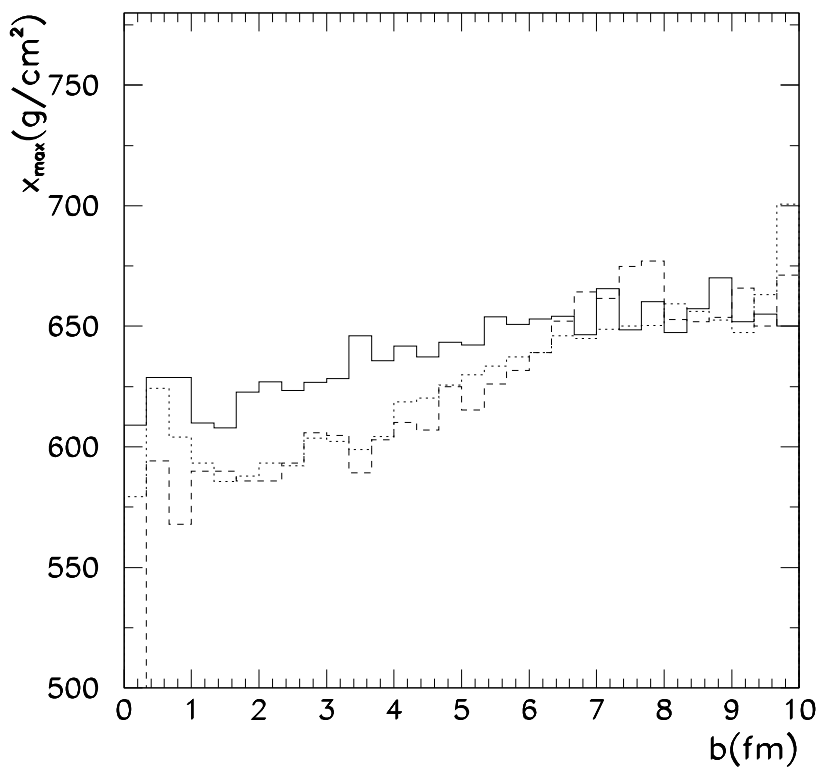

FIG. 8: Correlation between first-interaction impact parameter $b$ and shower maximum $X_{\max }$ for $10^{18} \mathrm{eV}$ iron initiated showers using the QGSJET model (continuous line) and the stopping power enhancement mechanism in the K1 case (dashed line) and K2 (dotted line).

first, string fusion 9] which strongly affects multiplicities but has very little effect on the forward region; second, a different picture of strong fields [11] which strongly increases the stopping. More concretely:

- We have implement the program PSM-1.0 which include collective effects like string fusion in a Monte Carlo that simulate air showers, AIRES. In this way we have shown that string fusion has almost no effect on air shower development because it only changes the multiplicity in the central region, the development of air showers being determined mainly by the particles in the forward region.

- We have shown that other collective effects like the increasing of the stopping power in the fragmentation region can have consequences in the shower development. The average $X_{\max }$ is moderately reduced; this faster shower development makes the shower look as initiated by a heavier primary. The distribution of muons at ground is reduced, thus mimicking a lighter primary in conventional scenarios. 


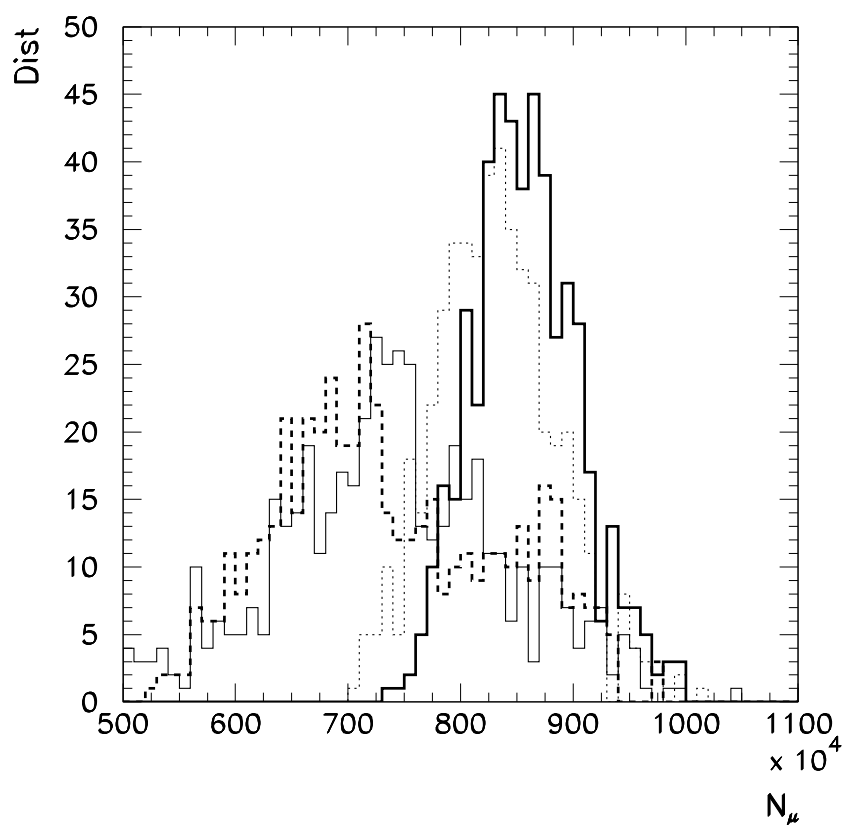

FIG. 9: Muon number distribution at ground for $10^{18} \mathrm{eV}$ iron initiated showers for standard QGSJET (thick continuous line), K1 (thick dashed line) and K2 (dotted line). Also shown the muon number distribution for proton initiated showers at the same energy (thin continuous line).

- Correlations between impact parameter and shower properties may arise in these models and constitute a novel aspect to be considered.

These effects may have important implications in the composition measurements and separation of proton-induced from iron-induced showers. They should also be taken into account to estimate the systematics of the primary energy determination based on shower profiles and number of muons at ground.

\section{ACKNOWLEDGMENTS}

We thank J. Álvarez-Muñiz, R. Engel, and E. Zas for suggestions and discussions. This work is supported by Xunta de Galicia (PGIDT00PXI20615PR), by CICYT (AEN99-0589C02-02), and by MCYT (FPA 2001-3837). We also thank the "Centro de Supercomputación 


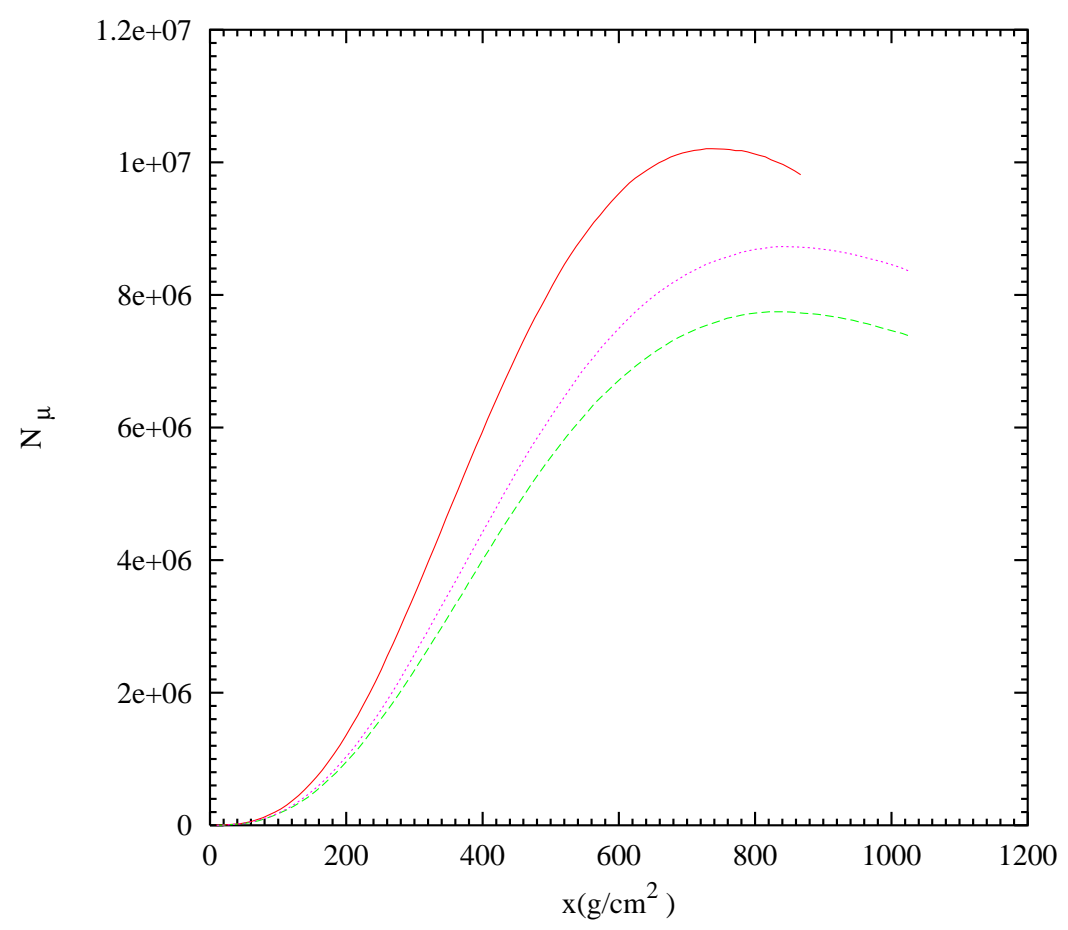

FIG. 10: Average $\mu^{+} \mu^{-}$longitudinal development for iron showers of $10^{18} \mathrm{eV}$ in standard QGSJET (continuous line), K1 (dashed line) and K2 (dotted line).

de Galicia” (CESGA) for computer resources.

[1] K. Greisen, Phys. Rev. Lett. 16, 748 (1966); G. T. Zatsepin and V. A. Kuzmin, JETP Lett. 4, 78 (1966) [Pisma Zh. Eksp. Teor. Fiz. 4, 114 (1966)].

[2] M. Nagano and A. A. Watson, Rev. Mod. Phys. 72, 689 (2000).

[3] R. Luna, A. Zepeda, C. A. García Canal, and S. J. Sciutto, arXiv:hep-ph/0408303.

[4] U. W. Heinz and M. Jacob, arXiv:nucl-th/0002042; M. Gyulassy and L. McLerran, arXiv:nucl-th/0405013.

[5] I. G. Bearden et al. [BRAHMS Collaboration], Phys. Rev. Lett. 93, 102301 (2004); B. B. Back et al. [PHOBOS Collaboration], arXiv:nucl-ex/0409003.

[6] N. Armesto, M. Braun, and C. Pajares, Phys. Rev. D 48, 162 (1993); Phys. Rev. C 51, 3393 (1995).

[7] C. Pajares, D. Sousa, and R. A. Vázquez, Phys. Rev. Lett. 86, 1674 (2001). 
[8] C. Pajares, D. Sousa, and R. A. Vázquez, Astropart. Phys. 12, 291 (2000).

[9] N. S. Amelin, M. A. Braun, and C. Pajares, Phys. Lett. B 306, 312 (1993); Z. Phys. C 63, 507 (1994).

[10] N. S. Amelin, N. Armesto, C. Pajares, and D. Sousa, Eur. Phys. J. C 22, 149 (2001);

N. Armesto, C. Pajares, and D. Sousa, Phys. Lett. B 527, 92 (2002).

[11] I. N. Mishustin and J. I. Kapusta, Phys. Rev. Lett. 88, 112501 (2002).

[12] V. N. Gribov, Sov. Phys. JETP 26, 414 (1968) [Zh. Eksp. Teor. Fiz. 53, 654 (1967)]; ibid. 29, 483 (1969) [56, $892(1969)]$.

[13] A. Capella, U. Sukhatme, C. I. Tan, and J. Tran Thanh Van, Phys. Rept. 236, 225 (1994); A. B. Kaidalov and K. A. Ter-Martirosyan, Phys. Lett. B 117, 247 (1982).

[14] N. N. Kalmykov, S. S. Ostapchenko, and A. I. Pavlov, Nucl. Phys. Proc. Suppl. 52B, 17 (1997).

[15] G. Bossard et al., Phys. Rev. D 63, 054030 (2001).

[16] J. Ranft, arXiv:hep-ph/9911232.

[17] V. A. Abramovsky, E. V. Gedalin, E. G. Gurvich, and O. V. Kancheli, Sov. J. Nucl. Phys. 53, 172 (1991) [Yad. Fiz. 53, 271 (1991)].

[18] N. Armesto, M. A. Braun, E. G. Ferreiro, C. Pajares, and Y. M. Shabelski, Astropart. Phys. 6, 327 (1997); Phys. Lett. B 389, 78 (1996).

[19] N. Armesto, M. A. Braun, E. G. Ferreiro, and C. Pajares, Phys. Rev. Lett. 77, 3736 (1996).

[20] M. A. Braun, F. Del Moral and C. Pajares, Phys. Rev. C 65, 024907 (2002); Eur. Phys. J. C 21, 557 (2001); M. A. Braun, C. Pajares, and J. Ranft, Int. J. Mod. Phys. A 14, 2689 (1999).

[21] S. J. Sciutto, arXiv:astro-ph/9905185, arXiv:astro-ph/9911331.

[22] J. Linsley and A. A. Watson, Phys. Rev. Lett. 46, 459 (1981).

[23] C. W. De Jager, H. De Vries, and C. De Vries, Atom. Data Nucl. Data Tabl. 14, 479 (1974). 